\title{
Trimetazidine and Bisoprolol to Treat Angina in Symptomatic Patients: Post Hoc Analysis From the CHOICE-2 Study
}

\author{
Maria Glezer · on behalf of the CHOICE-2 study investigators
}

Received: September 23, 2020 / Published online: November 27, 2020

(c) The Author(s) 2020

\section{ABSTRACT}

Introduction: Angina is the cardinal symptom of chronic coronary syndrome (CCS), which is the leading cause of death worldwide. As such, the control of angina is important. The current guidelines recommend beta blockers (BB) or calcium channel blockers to reduce angina, yet many patients with stable angina remain symptomatic. It has been suggested that combining trimetazidine (TMZ), an anti-ischemic agent, with a BB is beneficial for symptomatic patients. Bisoprolol, a $\mathrm{BB}$, is often used to treat patients with CCS, yet no data are currently available regarding the efficacy of bisoprolol combined with TMZ in patients who remain symptomatic despite receiving bisoprolol.

Methods: The aim of this post-hoc analysis of the CHOICE- 2 study was to evaluate the efficacy and safety of TMZ $35 \mathrm{mg}$ twice daily in combination with different bisoprolol doses in symptomatic patients with stable angina patients receiving hemodynamic therapy in a real-world clinical setting.

Results: This analysis involved 221 patients (mean [ \pm standard deviation] age $64.8 \pm 8.9$ years) with stable angina. The mean number of weekly angina episodes gradually fell from $6.2 \pm 5.3$ at inclusion (M0) to $1.5 \pm 1.9$ at 6 months after treatment initiation (M6) with combined TMZ-bisoprolol therapy $(P<0.001)$. The number of patients assessed to be anginafree increased almost sixfold from 5.4\% (12/ $221)$ at M0 to $33.9 \%(74 / 221)$ at M6. Exercise capacity improved, as measured by walking distance, from $308 \pm 207 \mathrm{~m}$ at M0 to $497 \pm 253 \mathrm{~m}$ at M6 $(P<0.05)$. The number of patients with Canadian Cardiovascular Society class 1 angina increased by tenfold during the study, whereas those with class 3 angina decreased by threefold.

Conclusion: The TMZ-bisoprolol combination is a rapidly effective treatment for reducing the frequency of angina attacks and the use of short-acting nitrates in patients with stable angina in a real-world clinical setting. The benefits of this combination therapy was observed as early as 2 weeks after treatment initiation and the treatment was well tolerated. Trial Registration: ISRCTN identifier: ISRCTN65209863

Keywords: Angina; Bisoprolol; Real-world clinical setting; Trimetazidine
M. Glezer ( $\square)$

Department of Cardiology, Functional and

Ultrasound Diagnostics, Sechenov First Moscow

State Medical University, Moscow, Russia

e-mail: 287ast@mail.ru 


\section{Key Summary Points}

Why carry out this study?

Angina remains a therapeutic problem in current real-life medical practice despite current conventional first-line hemodynamic anti angina therapies.

Bisoprolol is one of the most widely used beta blockers (BB) for the relief of angina in patients with chronic coronary syndrome.

Trimetazidine (TMZ), an anti-ischemic agent, has demonstrated additional angina relief in patients who remain symptomatic while on $\mathrm{BB}$, but data regarding its efficacy when used in combination with bisoprolol are scarce.

\section{What was learned from the study?}

The current analysis presents real-world data on the anti-angina efficacy of TMZ $35 \mathrm{mg}$ twice daily when included in the therapeutic regimen of patients with angina who have remained symptomatic despite treatment with maximum tolerated bisoprolol dose.

\section{DIGITAL FEATURES}

This article is published with digital features to facilitate understanding of the article. You can access the digital features on the article's associated Figshare page. To view digital features for this article go to https://doi.org/10.6084/m9. figshare.13160387

\section{INTRODUCTION}

Chronic coronary syndrome (CCS) is currently the leading cause of death worldwide [1]. Angina, the cardinal symptom of CCS, often requires hospitalization and substantial resource use $[2,3]$. As survival and major cardiac

events are closely related to the frequency of angina attacks, alleviating symptoms is one of the main treatment goals for patients with CCS. Thus, antianginal (AA) drugs are a fundamental part of the therapy to relieve chest pain and improve quality of life. Both the European and American guidelines recommend beta blockers (BB) or calcium channel blockers (CCB) in combination or alone as first-line treatment for symptomatic angina relief [4]. Worldwide registry evidence demonstrates that these recommendations are well followed as BB either as monotherapy or in combination with other AA drugs are the most widely used drugs in realworld clinical practice to alleviate angina in patients with CCS [5]. However, despite this widespread use of $\mathrm{BB}$, a high proportion of patients with stable angina continue to experience ischemia and anginal pain [6], even after successful revascularization [7-9]. An analysis of patient data in CLARIFY, the largest registry of patients with CCS, demonstrated that $79 \%$ of the patients who complain of angina were treated with a $\mathrm{BB}$, among which bisoprolol was the most frequently prescribed $\mathrm{BB}$ [10].

Trimetazidine (TMZ) is a metabolic agent that acts at the cellular level, optimizing glucose metabolism (adenosine triphosphate) and in doing so, protects against angina and ischemic damage $[11,12]$. During an ischemic attack, TMZ has a cytoprotective effect and is thus indicated in the long-term treatment of stable angina. Its effects complement those of hemodynamically active agents and reduce the frequency of angina episodes without affecting the hemodynamic parameters [13, 14]. This cardioprotective effect has been shown both in patients with either CCS [14-18] and those with heart failure [19-22]. There is also some evidence suggesting that some patients remain symptomatic despite taking BB, such as propranolol or metoprolol, and would gain benefit from adding TMZ to their background BB therapy $[23,24]$.

We report here a post-hoc analysis of the CHOICE-2 study in which we evaluated the efficacy and safety of TMZ $35 \mathrm{mg}$ twice daily (bid) added to bisoprolol in patients with persisting angina despite taking the maximal tolerated bisoprolol dose and other appropriate 
background hemodynamic therapy, in realworld clinical practice.

\section{METHODS}

The design and conduct of this observational, multicenter study have been described in detail in a previous publication on the CHOICE II study [25].

CHOICE-2 was a 6-month multicenter observational program conducted in 46 regions of the Russian Federation (ISRCTN identifier: ISRCTN65209863, protocol number IC406,790-013-RUS) [25]. All patients were treated with modified-release TMZ (TMZ MR) $35 \mathrm{mg}$ bid in combination with a $\mathrm{BB}$ in addition to background hemodynamic therapy. The study enrolled 896 patients with stable angina, the majority of whom were classified with Canadian Cardiovascular Society class 2 or 3 angina and were being treated with a maximum tolerated dose of a BB, either alone or in combination with another first or second class AA drug. The treating general practitioner or cardiologist was free to decide whether TMZ MR $35 \mathrm{mg}$ bid could be added to the current AA therpeutic regimen. Physicians were also free to modify the AA drugs or implement secondary prevention during study visits, if deemed necessary according to their clinical judgment and normal clinical practice. Over the course of the study, safety and efficacy parameters were assessed at five visits (inclusion [M0]; week 2 [W2] and months 2, 4 and 6 (M2, M4 and M6, respectively, after treatment initiation).

All procedures comply with the ethical standards of the responsible committee on human experimentation (institutional and national), the Helsinki Declaration of 1964, as revised in 2013, and the European Independent Ethics Committee. The CHOICE-2 protocol was approved by the Inter-University Ethics Committee (protocol no. 09-14 dd. 23/10/2014; Moscow), and informed consent for inclusion in the study was obtained from all patients.

The current post-hoc analysis was performed because there are currently no data on the efficacy of TMZ when added to bisoprolol monotherapy in patients with CCS who remain symptomatic. The analysis was performed on a subset of patients receiving combination therapy with TMZ + bisoprolol in addition to other hemodynamic therapy. The patients consistently received the maximally tolerated dose of bisoprolol, and this regimen remained unchanged 1 month before inclusion in the study. Other hemodynamically active agents were allowed throughout the study according to the investigator's discretion.

Of the 896 patients enrolled in the CHOICE II study, 221 patients were treated with both TMZ $35 \mathrm{mg}$ bid and bisoprolol once daily throughout the study period. For the purposes of this analysis, patients were categorized into four groups based on the prescribed bisoprolol dose: $2.5 \mathrm{mg}, 5 \mathrm{mg}, 10 \mathrm{mg}$ or Other doses. Safety and efficacy data from the five study visits were included in the post hoc analysis. For each bisoprolol dose level and for the whole population treated with the combination of bisoprolol $+\mathrm{TMZ}$, the investigator recorded the weekly number of angina attacks, weekly consumption of short-acting nitrates (SAN), walking distance, stair climbing before chest pain onset, presence of dyspnoea and echocardiography parameters. The Canadian Cardiovascular Society functional classification system was used to assess angina severity.

The only safety measurements included in the analysis were those of systolic and diastolic blood pressure (SBP and DBP, respectively, in $\mathrm{mmHg}$ ), heart rate (bpm) and clinical laboratory evaluations (total cholesterol, high-density lipoproteins, low-density lipoproteins, triglycerides, fasting blood glucose, glycosylated hemoglobin, creatinine, uric acid). In addition, any hospitalization or ambulance call-out for these patients was recorded.

All of the parameters were analyzed using methods for descriptive statistics. For each parameter, the number of patients, mean value, standard error, and minimal and maximal values were presented. All outcomes, including baseline characteristics and safety, were analyzed in the intention-to-treat (ITT) population. Changes in parameters (and confidence intervals) were evaluated in the "per protocol" population (PPP). For the analysis of normally distributed data, the Student's $t$ test for paired 
measurements was used; otherwise, the nonparametrical Wilcoxon test was used. Additional pharmacodynamic parameters were assessed by discriminative analysis.

\section{RESULTS}

\section{Population}

A total of 221 patients were included in the analysis. The demographic and baseline characteristics of the patient population are listed in Table 1 . The population was relatively young, with a mean ( \pm standard deviation $[S D]$ ) age of $64.8 \pm 8.9$ years, and the proportion of men $(47.96 \%)$ to women was well-balanced in the total population; however there were more men in $2.5 \mathrm{mg}$ bisoprolol group (69.23\%) and in the 'Other' bisoprolol group (60\%). Blood pressure was relatively well-controlled in the total population (mean \pm SD: SBP $139.4 \pm 15.9 \mathrm{mmHg}$, DBP: $84.4 \pm 9.7 \mathrm{mmHg}$ ). Mean heart rate was $71.9 \pm 9.6 \mathrm{bpm}$ which while being within acceptable range, was on the border of high. Only 25\% were former smokers or had never smoked. Most patients had hypertension (91.86\%) and nearly one-quarter (23.08\%) had diabetes; (22.62\%) reported having had a coronary revascularization. All patients had stable angina at inclusion, with most of them classified as grade $2(65.6 \%)$ or grade $3(30.8 \%)$. The mean number $( \pm \mathrm{SD})$ of angina episodes was $6.2 \pm 5.3$ (range 2-38) episodes per week, and the mean number of SAN intakes per week was $5.5 \pm 4.4$ (range 0-25). All patients were taking a $\mathrm{BB}, 25.4 \%$ were taking a $\mathrm{CCB}$ and $26.7 \%$ were also taking long-acting nitrates.

All patients were prescribed TMZ $35 \mathrm{mg}$ bid throughout the study. A majority of patients (60.1\%) were prescribed $5 \mathrm{mg}$ bisoprolol; $21.1 \%$ were prescribed $10 \mathrm{mg}$ and $11.9 \%$ received $2.5 \mathrm{mg}$ bisoprolol. In the patient group of Other bisoprolol doses, $1.8 \%$ of patients were treated with a dose of $7.5 \mathrm{mg}, 0.9 \%$ with a dose of $1.25 \mathrm{mg}$, and $0.5 \%$ with a dose with $15 \mathrm{mg}$. Of the 221 patients, $92.8 \%$ remained on the same bisoprolol dose throughout the trial and 218 remained on the TMZ-bisoprolol combination after the study.
During the study period, AA drugs and other cardiovascular therapy remained stable (rate of change per treatment $<5 \%$ ). The use of AA therapy fell slightly from inclusion (M0) to M6; this trend was also seen for CCB use, from 25 to $24 \%$, and for use of long-acting nitrates, 27 to $20 \%$.

\section{Outcome Results}

All outcomes, including safety, were analyzed in the 221 patients in the ITT population.

\section{Angina Episodes}

The mean number $( \pm S D)$ of reported angina episodes per week decreased progressively from $6.2 \pm 5.3$ at inclusion (M0) to $4.4 \pm 4.0$ at W2, and continued decreasing to $1.5 \pm 1.9$ at M6. Mean change from baseline was statistically significant at all evaluated time points $(P<0.001)$.

Similar decreases were observed when each bisoprolol subgroup was considered separately. The mean number of reported angina attacks per week decreased significantly and sustainably from inclusion (M0) to M6 in all four subgroups $(P<0.05)$ (Fig. 1)

\section{Proportion of Angina-Free Patients}

At inclusion (M0), all patients reported at least one angina episode per week. In patients prescribed the combined TMZ-bisoprolol, the percentage (number) of angina-free patients steadily increased by almost sixfold, from $5.4 \%$ $(12 / 221)$ at inclusion to $33.9 \%(74 / 221)$ at M6. A similar trend of angina-free patients was observed in the bisoprolol dosage subgroups (Fig. 2).

\section{Use of Short-Acting Nitrates}

Overall, the mean number $( \pm S D)$ of reported SAN doses per week steadily decreased from $5.5 \pm 4.4$ at inclusion (M0) to $1.0 \pm 1.4$ at M6. Mean changes from baseline were statistically significant at all evaluated time points 
Table 1 Baseline characteristics of patient population

\begin{tabular}{|c|c|c|c|c|c|}
\hline \multirow{2}{*}{$\begin{array}{l}\text { Patient baseline } \\
\text { characteristics }\end{array}$} & \multicolumn{4}{|c|}{ Patient groups based on prescribed bisoprolol dose } & \multirow{2}{*}{$\begin{array}{l}\text { All patients } \\
(n=221)\end{array}$} \\
\hline & $\begin{array}{l}2.5 \mathrm{mg} \\
(n=26)\end{array}$ & $\begin{array}{l}5 \mathrm{mg} \\
(n=140)\end{array}$ & $\begin{array}{l}10 \mathrm{mg} \\
(n=45)\end{array}$ & $\begin{array}{l}\text { Other } \\
(n=10)\end{array}$ & \\
\hline Age (years) & $67.8 \pm 8.1$ & $65.0 \pm 8.6$ & $62.5 \pm 9.3$ & $64.8 \pm 8.8$ & $64.8 \pm 8.9$ \\
\hline Men, $n(\%)$ & $18(69.23)$ & $64(45.71)$ & $18(40)$ & $6(60)$ & $106(47.96)$ \\
\hline BMI $\left(\mathrm{kg} / \mathrm{m}^{2}\right)$ & $29.1 \pm 4.6$ & $30.7 \pm 4.7$ & $30.4 \pm 5.0$ & $28.9 \pm 3.5$ & $30.4 \pm 4.7$ \\
\hline $\begin{array}{l}\text { Current \& former smokers } \\
n(\%)\end{array}$ & $85(30.76)$ & $39(27.85)$ & $9(20)$ & $0(0)$ & $56(25.34)$ \\
\hline $\mathrm{SBP}(\mathrm{mmHg})( \pm \mathrm{SD} 11)$ & $133.7 \pm 18.0$ & $139.6 \pm 15.6$ & $143.4 \pm 15.0$ & $132.8 \pm 12.9$ & $139.4 \pm 15.9$ \\
\hline $\mathrm{DBP}(\mathrm{mmHg})( \pm \mathrm{SD} 12)$ & $79.2 \pm 10.8$ & $84.7 \pm 9.5$ & $86.9 \pm 8.9$ & $81.7 \pm 7.5$ & $84.4 \pm 9.7$ \\
\hline Heart rate $(\mathrm{bpm})$ & $69.4 \pm 10.1$ & $72.8 \pm 9.3$ & $72.1 \pm 10.3$ & $66.4 \pm 7.2$ & $71.9 \pm 9.6$ \\
\hline \multicolumn{6}{|l|}{ Concomitant diseases, $n(\%)$} \\
\hline Hypertension & $21(80.77)$ & $131(93.57)$ & $43(95.56)$ & $8(80.00)$ & $203(91.86)$ \\
\hline $\begin{array}{l}\text { Previous myocardial } \\
\text { infarction }\end{array}$ & $12(46.2)$ & $54(38.57)$ & $15(33.33)$ & $7(70.00)$ & $88(3.82)$ \\
\hline Diabetes, & $3(11.54)$ & $32(22.86)$ & $13(28.89)$ & $3(30.00)$ & $51(23.08)$ \\
\hline Previous revascularization & $6(23.08)$ & $31(22.14)$ & $10(22.22)$ & $3(30.00)$ & $50(22.62)$ \\
\hline Respiratory disease & $0(0.00)$ & $17(12.14)$ & $4(8.89)$ & $0(0.00)$ & $21(9.50)$ \\
\hline Chronic kidney disease & $6(23.08)$ & $24(17.14)$ & $6(13.33)$ & $2(20.00)$ & $38(17.19)$ \\
\hline \multicolumn{6}{|c|}{ Treatments taken at inclusion, $n(\%)$} \\
\hline beta-blocker & $26(100)$ & $140(100)$ & $45(100)$ & $10(100)$ & $221(100)$ \\
\hline Calcium antagonists & $5(19.23)$ & $33(23.57)$ & $15(33.33)$ & $3(30.00)$ & $56(25.34)$ \\
\hline Long-acting nitrates & $3(11.54)$ & $34(24.29)$ & $19(42.22)$ & $3(30.00)$ & $59(26.70)$ \\
\hline ACE inhibitors or sartans & $22(84.62)$ & $123(87.86)$ & $43(95.56)$ & $8(80.00)$ & $196(88.69)$ \\
\hline Diuretics & $11(42.31)$ & $73(52.14)$ & $31(68.89)$ & $6(60.00)$ & $121(54.75)$ \\
\hline Anti-platelet agents & $25(96.2)$ & $134(95.7)$ & $45(100.0)$ & $9(90.00)$ & $213(96.38)$ \\
\hline Anticoagulants & $1(3.85)$ & $6(4.29)$ & $0(0.00)$ & $1(10.00)$ & $8(3.62)$ \\
\hline Statins & $22(84.62)$ & $132(94.29)$ & $43(95.56)$ & $10(100.0)$ & $207(93.67)$ \\
\hline
\end{tabular}

Values in table are presented as the mean \pm standard deviation or a number with the percentage in parentheses, as appropriate

$A C E$ Angiontensin-converting enzyme, $B M I$ body mass index, $D B P$ diastolic blood pressure, $S B P$ systolic blood pressure, $S D$ standard error 


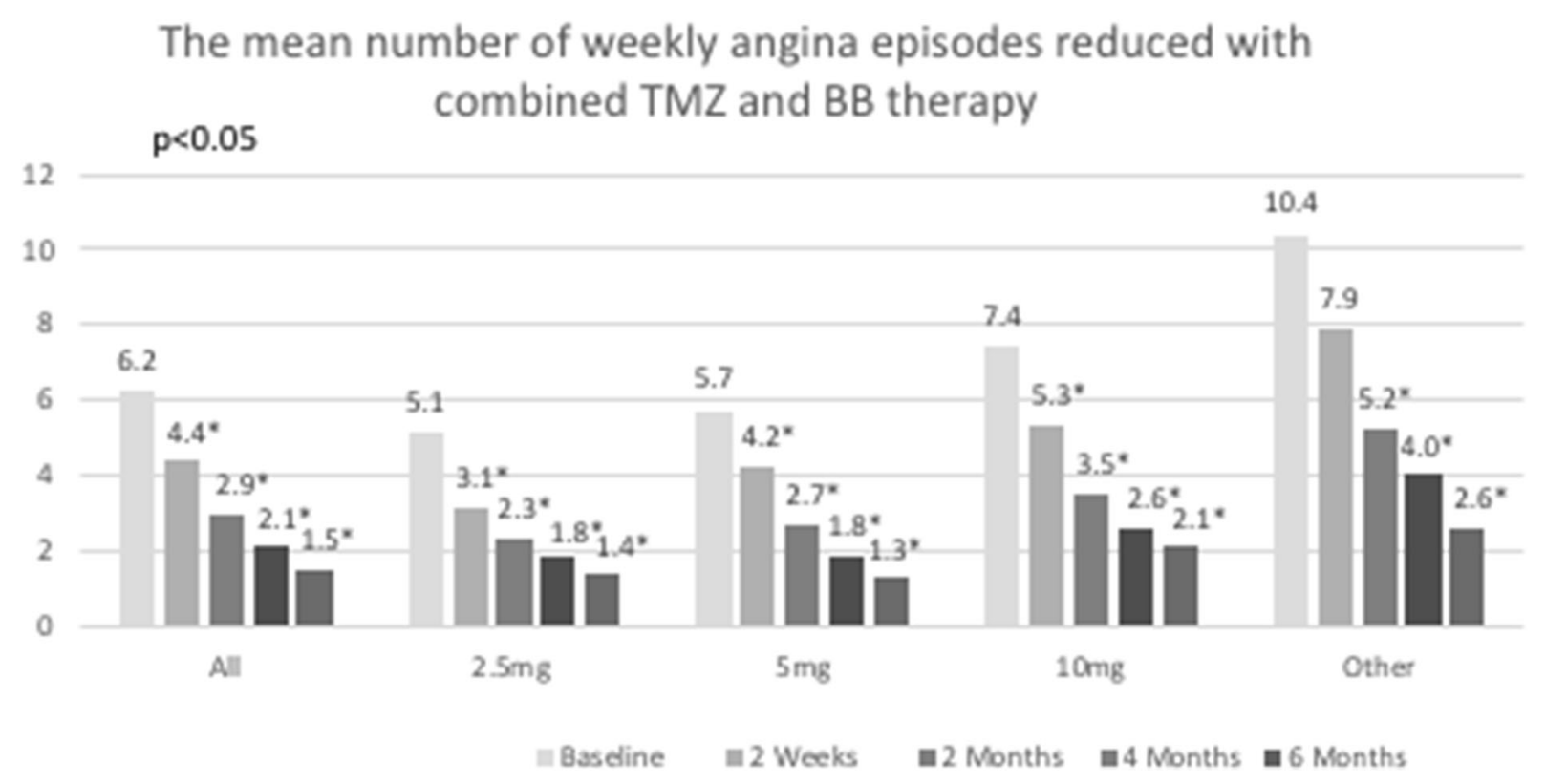

Fig. 1 The mean number of reported angina episodes from baseline $(M O)$ to 6 months after initiation of treatment $(M \sigma)$ with trimetazidine (TMZ) added to there is a steady decrease in the number of reported angina episodes with increasing duration of treatment regimen of patient-adapted bisoprolol doses. Note that

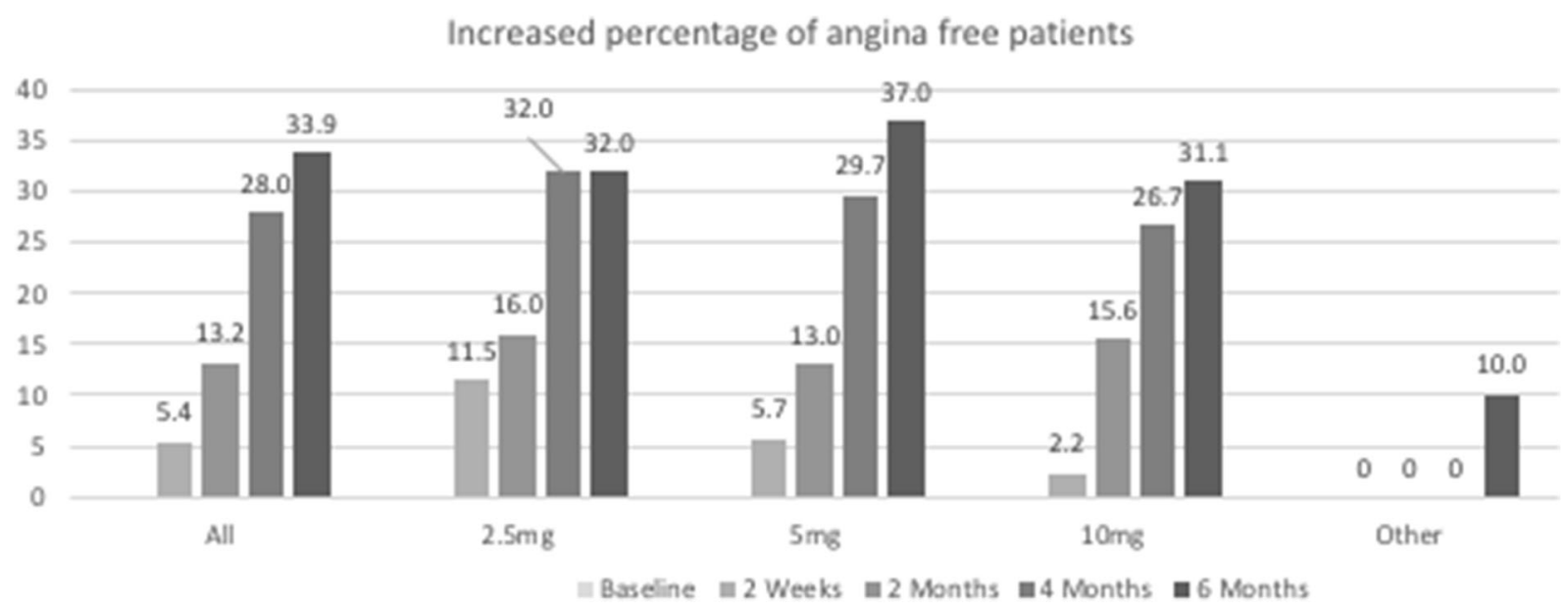

Fig. 2 Proportion of angina-free patients from inclusion $(M O)$ to 6 months after treatment initiation $(M \sigma)$ with combined TMZ-bisoprolol therapy. Note that the proportion of angina-free patients increases from baseline to M6

$(P<0.001)$ : W2 $(-1.9 \pm 2.9), \mathrm{M} 2(-3.2 \pm 3.0)$, M4 $(-4 \pm 3.5)$ and M6 ( $4.5 \pm 3.7)$. Similar decreases were observed when each bisoprolol dose subgroup was considered separately. The mean number of reported SAN doses per week decreased significantly from inclusion to M6 in all four subgroups $(P<0.05)$.
During the 6-month treatment period, the percentage (number) of patients who no longer required SAN steadily increased, from 5.9\% (13/ $221)$ at inclusion to $52.3 \%(114 / 218)$ at M6 (Fig. 3). 


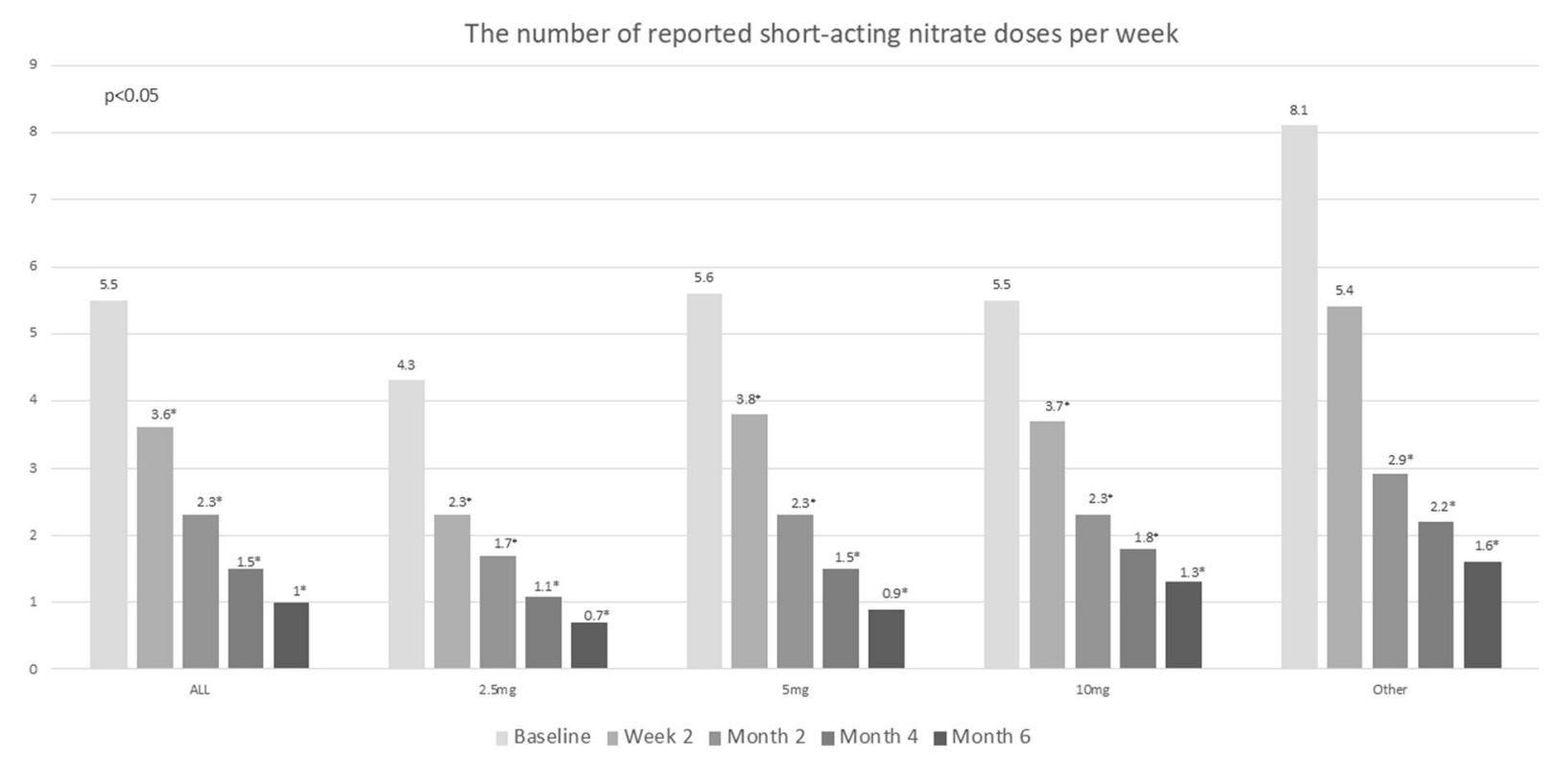

Fig. 3 Mean changes in weekly short-acting nitrate $(S A N)$ doses from inclusion $(M O)$ to 6 months after treatment initiation (M6) with combined TMZ-bisoprolol therapy.

\section{Walking Distance to Elicit Chest Pain}

The mean $( \pm S D)$ walking distance to elicit chest pain steadily increased in all bisoprolol dose subgroups, from $308 \pm 207 \mathrm{~m}$ at inclusion to $497 \pm 253 \mathrm{~m}$ at 6 months $(P<0.05)$. Mean changes from baseline were statistically significant at all of the evaluated time points $(P<0.05): \quad$ W2 $\quad(+23 \pm 131 \mathrm{~m}), \quad$ Me $(+89 \pm 168 \mathrm{~m}), \mathrm{M} 4(+138 \pm 145 \mathrm{~m})$ and M6 $(+189 \pm 169 \mathrm{~m})$ (Fig. 4).

\section{Stair Climbing Before Chest Pain Occurred}

The mean number $( \pm$ SD) of flights climbed before chest pain occurred steadily increased from $2.9 \pm 2.0$ at inclusion to $4.5 \pm 2.3$ at M6. The results for the whole study population were reflected in all four subgroups, with a significant increase in the number of flights climbed before chest pain occurred from inclusion to M6 $(P<0.05)$. Mean changes from baseline were statistically significant at all visits after W2 $(P<0.001)$.
Note that the number of mean SAN doses decreases from inclusion to M6

\section{Dyspnoea}

Overall, the percentage (number) of patients presenting dyspnoea steadily decreased from inclusion to M6 (76.5\% [169/221] to 39.4\% [87/ 221]). The results for dyspnoea per subgroup were consistent with those for the whole study population, all indicated a lower frequency of dyspnoea over time.

\section{Angina Severity}

Angina severity was classified according to the Canadian Cardiovascular Society functional classification system as class $1,2,3$ or 4 . The percentage of patients with class 1 angina increased more than tenfold during the study (from $2.3 \%$ at inclusion to $27.6 \%$ at M6), while the percentage of patients with class 3 angina decreased threefold (from $30.8 \%$ at inclusion to $10.0 \%$ at M6) (Fig. 5).

Changes in angina severity were also classified as: stable (no shift in angina severity grade from inclusion to M6); improving (shift towards a lower severity grade from inclusion to M6); and worsening (shift towards a higher severity 


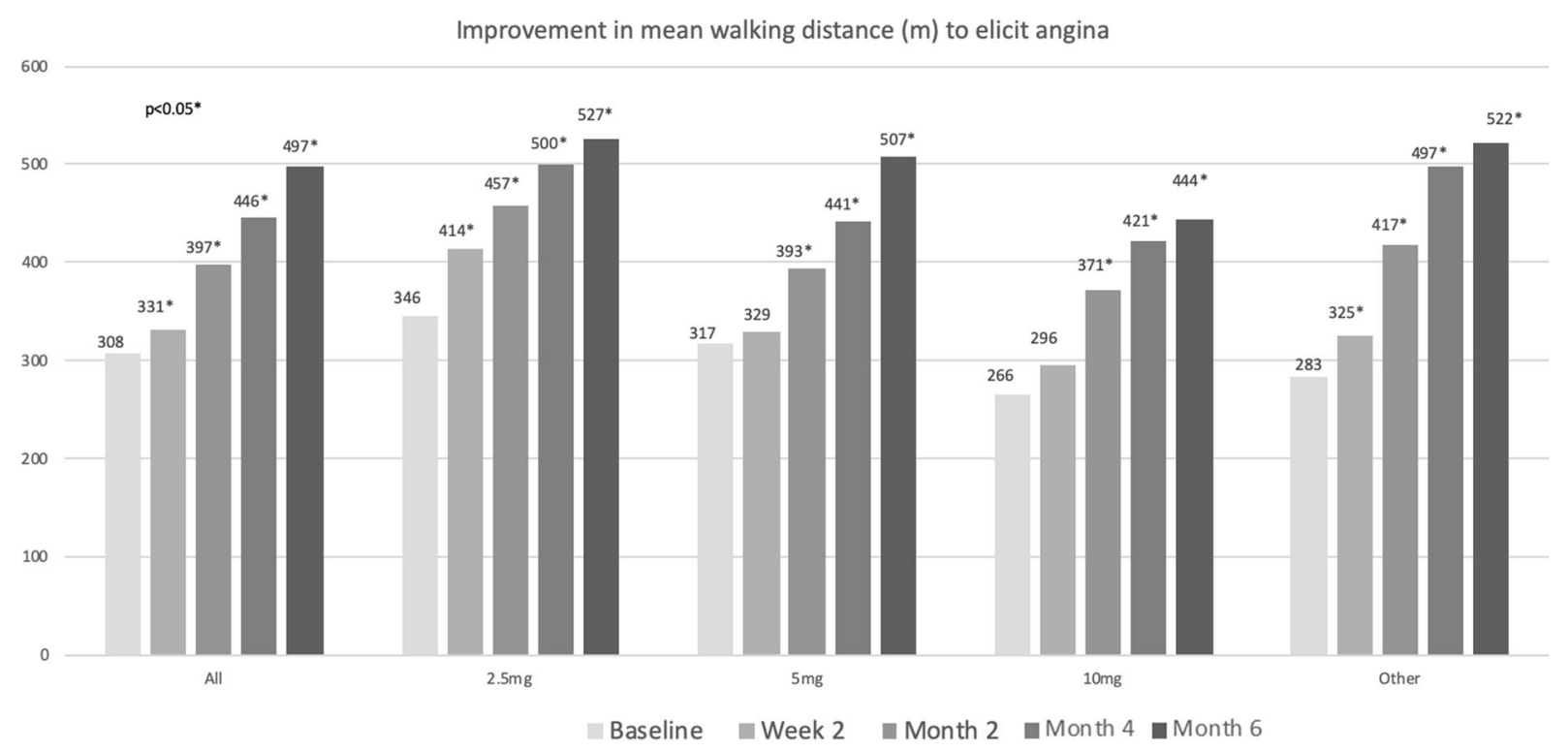

Fig. 4 Mean changes in walking distance from inclusion (MO) to 6 months after treatment initiation (M6) with combined TMZ-bisoprolol therapy. Note that the mean walking distance increased from inclusion to M6

\section{Improvement in angina severity}

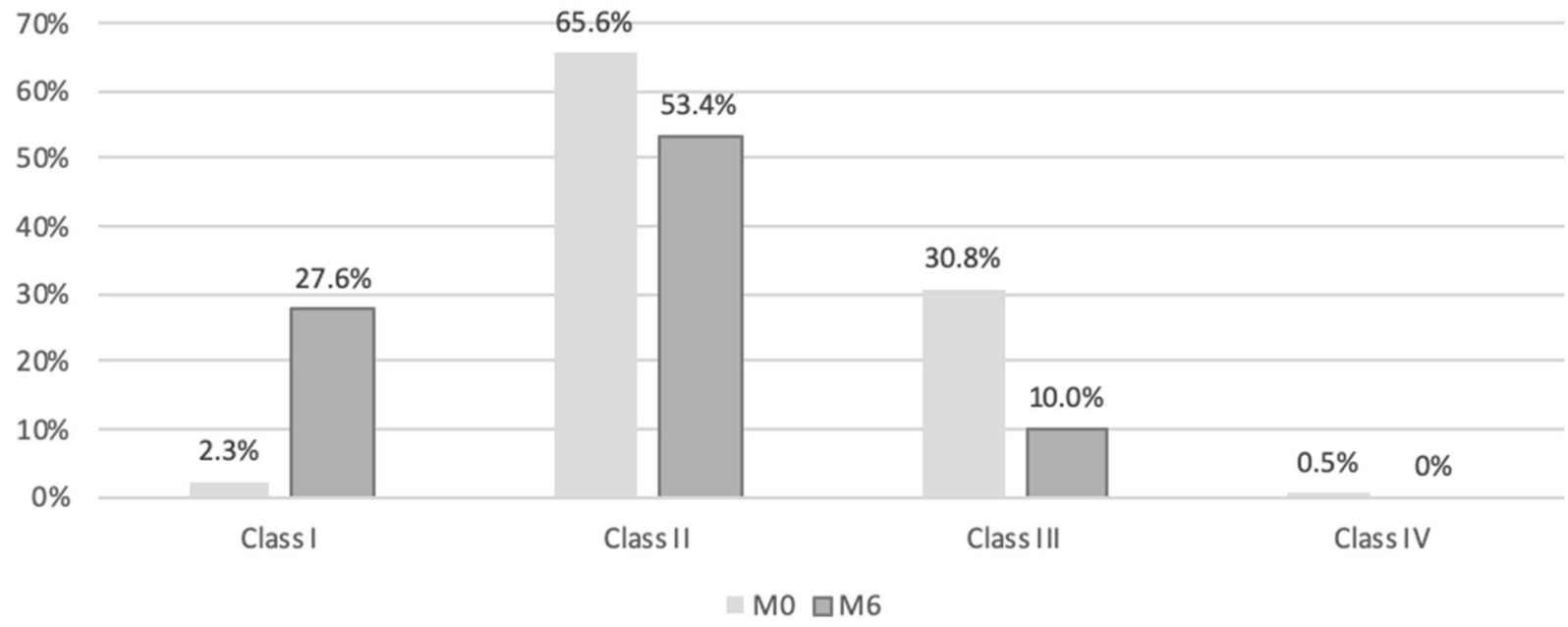

Fig. 5 Change in angina severity according to the the Canadian Cardiovascular Society functional classification system from inclusion $(M O)$ to 6 months after treatment initiation (M6) with combined TMZ-bisoprolol therapy.

grade from inclusion to M6). Of the 221 patients included in the full analysis set, two (0.9\%) showed worsening angina severity, 96 (43.4\%) showed improvement in angina severity and $101(45.7 \%)$ showed neither improving nor worsening of their angina symptoms;
Note that addition of TMZ to the treatment regimen reduced angina severity at M6

changes in angina severity could not be calculated for the remaining 22 patients (10.0\%). 


\section{Echocardiographic Parameters}

The mean ejection fraction was $57.2 \pm 7.7 \%$ $(n=136)$ at inclusion and $57.3 \pm 7.9 \%(n=43)$ at M6. The mean change from baseline could only be calculated for 33 patients and was $1.5 \pm 3.6 \%$.

There were no significant changes in echocardiographic parameters (left ventricular posterior wall, interventricular septum) after 6 months of treatment with the TMZ-bisoprolol combination.

\section{Safety Results}

During the study, blood pressure remained within normal ranges in all subgroups. No bradycardia was observed. No deterioration was observed in the lipid, glucose, or uric acid profiles.

Most hospitalizations and ambulance calls were related to angina and concomitant diseases such as hypertension. The number of hospitalizations and ambulance calls decreased from 72 (reported by 61/221 patients) in the 6 months preceding study inclusion to 23 (reported by 17 patients) during the treatment period. This threefold decrease in the hospitalization rate was consistently observed in the TMZ-bisoprolol subgroups.

\section{DISCUSSION}

The current analysis provides real-world evidence in support of the hypothesis that the addition of TMZ $35 \mathrm{mg}$ bid to bisoprolol monotherapy at various bisoprolol doses provides additional benefits to patients with symptomatic angina on hemodynamic therapy, specifically the improvement of symptoms and physical capacity and reduction of SAN consumption.

BB are a heterogeneous class of drugs that differ in their B-receptor selectivity, their intrinsic sympathomimetic activity and their pharmacokinetic properties [26, 27]. Therefore, the choice of $\mathrm{BB}$ is important and is dependent on patient treatment goals, co-morbidities and tolerability.

We observed no changes in the glucose, lipid and uric acid profiles, bradycardia or hypotension in patient receiving the bisoprolol $+\mathrm{TMZ}$ combination treatment. Only one patient in the $5 \mathrm{mg}$ bisoprolol group changed therapy to metoprolol while hospitalized due to myocardial infarction; $98.6 \%$ of patients were advised by their physician to continue bisoprolol treatment after the study. These results support evidence from a recent meta-analysis in the UK of $>7000$ newly diagnosed outpatients with angina and co-morbid conditions who were followed for up to 14 years. In addition to bisoprolol demonstrating a probable superiority for various cardiovascular events, including angina, over other BB, it was associated with a higher adherence [28]. In fact, $90.6 \%$ of patients in the bisoprolol cohort were $\geq 80 \%$ adherent to their medication compared with only $63.2 \%$ of patients in the cohort receiving another BB [28].

In our study, the treating physician chose patients who were already being treated with the maximum tolerated dose of the BB, according to current treatment recommendations for stable angina, to alleviate symptoms [29]. Most patients (60.1\%) enrolled in the study were receiving $5 \mathrm{mg}$ bisoprolol and yet remained symptomatic, thus requiring further treatment optimization, despite having other AA drugs in their treatment regimen. Also, the mean heart rate bordered on the high side although it was within an acceptable range $(71.9 \pm 9.6 \mathrm{bpm})$. These findings reflect those of the CLARIFY registry study, which demonstrated that the maximum BB dose is often not reached in clinical practice. This registry study found that CCS patients were prescribed a BB dose that was one-half of that used in clinical studies [30], often because higher BB dosages increase side effects without a relevant efficacy, thereby reducing patient adherence [31, 32].

Current guidelines acknowledge the multifactorial origin of angina and encourage the use of more than one AA drug to alleviate angina pain $[5,29]$. To date, there are no data available to support superiority between the different AA classes. Moreover, the most recent CCS guidelines even foster early use of a combination of 
first- and second-line AA drugs to alleviate angina quickly, especially in patients who cannot tolerate the intense first-line regimen $[5,29]$. However, optimization of therapy with two to three drugs that affect similar hemodynamic mechanisms that cause ischemia often has no additive effect. These combinations often fail to provide effective angina relief, with the result that a vast number of patients remain symptomatic. In the ORBITA study, for example, AA treatment was optimized with two or more hemodynamic drugs for 6 weeks prior to randomization for percutaneous coronary intervention (PCI), yet among the 230 patients enrolled, symptoms regressed in only 17 patients [33].

TMZ acts at the cellular level and has no effect on coronary flow, contractility, blood pressure or heart rate and has been proven to be as effective as other non-heart rate-reducing AA drugs and heart rate-reducing drugs [17].

A recent meta-analysis that included 13 studies, five of which with a Jadad score of 4 and 5, involving 1628 patients with CCS patients demonstrated that combining TMZ or rimetazidine with the first-line AA therapy in patients still having symptoms is highly effective in alleviating angina regardless of the duration of treatment [34]. Moreover, combining BB with TMZ has proven to be more effective than combining the same BB with other AA drugs, such as long-acting nitrates [23]. It should be noted that the results of the well-controlled ATPCI study were published as this paper was submitted for publication. However, the AA properties of TMZ in patients included in our subanalysis of the CHOICE II study cannot be compared to those of patients in the ATPCI study as the patient populations are very different. The ATPCI study did not include angina patients by design and so was not powered to show AA efficacy for TMZ. Instead, the ATPCI study included patients following successful, uncomplicated PCI who were almost asymptomatic (mean Seattle Angina Questionnaire subdomain Angina Frequency [SAQ AF] score 91). The patients in the ATPCI study actually had a much lower angina burden than cohorts in similar studies $[9,35,36]$, which made it extremely challenging to prove additional benefits, particularly as the study was not powered to evaluate AA efficacy.

It can therefore be speculated that angina and functional status improvements in symptomatic patients on bisoprolol in our study were due to TMZ as all patients were taking the maximum tolerated dose of bisoprolol at inclusion, but remained symptomatic. Almost 93\% of patients remained on the initial bisoprolol dose throughout the study period, and no substantial hemodynamic treatment changes were made.

In addition, the AA efficacy of the combined TMZ-bisoprolol regimen was observed at as early as W2, fitting with the current 2019 European Society of Cardiology (ESC) guidelines for CCS that recommend symptom alleviation within 2-4 weeks from treatment initiation. During the study we noted a trend in reduced long-acting nitrates, from $27 \%$ at baseline to $20 \%$ at M6, which might be due to the improved angina status in those patients on the combined TMZ-bisoprolol regimen. Additionally, in our study, re-hospitalizations and ambulance calls mostly linked to CCS/angina or other co-morbidities, or to treatment side effects, consistently decreased in all dose groups until M6, compared to baseline, which underlines not only the efficacy but also the excellent safety profile of TMZ, which has no effect on the heart rate, blood pressure or glucose and lipid profiles. In the study, mean blood pressure reached $126 \pm 9.3 / 77 \pm 6.5 \mathrm{mmHg}$, which is especially important because there is a J curve relationship between blood pressure and angina incidence. When the DBP is $<70 \mathrm{mmHg}$, the frequency and severity of angina increases; when the SBP/DBP is $<120 / 70$, the frequency of death and myocardial infarction increases $[37,38]$.

In our study, only $1.67 \%$ of patients discontinued TMZ [25]. The current 2019 ESC guidelines for CCS recommend that AA therapy be tailored to the patient, the patient's preferences, comorbidities and potential drug interactions. Patients with angina are often co-morbid [6] and take many medications, so safety and tolerability of the AA therapy are as important as efficacy. 
According to the 2019 ESC guidelines for CCS, optimal medical therapy is defined as effective symptom alleviation with the fewest possible side effects and maximum treatment adherence. Early treatment with a combination of first- with second-line drugs is recommended taking into account the multifactorial origin of angina, the lack of evidence on the superiority of the AA class of drugs and the need for fast relief, along with acceptable tolerability, especially in certain patient populations. The results of our analysis demonstrate that combining bisoprolol with TMZ meets these criteria very rapidly (as early as week 2 after treatment initiation) and that sustained AA efficacy is provided with an excellent tolerability profile.

\section{Limitation of This Post-hoc Analysis}

For the most part, this post-hoc analysis is subject to the real-world limitations associated with the open-label, observational, non-interventional design of the original CHOICE-2 study [25]. In addition, the separate analysis of the subset of patients taking bisoprolol concomitantly with TMZ was not pre-planned and no sample size calculation was conducted to determine the optimal number of patients needed to determine a statistically significant result. The only data available were those from the original CHOICE-2 study, for which only the ongoing status of concomitant cardiovascular treatments was reported at each visit. Dates of bisoprolol intake were not recorded. As a result, it is not possible to describe accurately the extent of exposure to bisoprolol treatment in patients.

\section{CONCLUSIONS}

This post-hoc analysis shows that the combination therapy with TMZ + bisoprolol is a rapid and effective treatment in the real-world clinical setting for reducing the frequency of both angina attacks and SAN use in patients with stable angina. The TMZ-bisoprolol combination improved patient exercise capacity and was well tolerated in this post-hoc analysis population.

\section{ACKNOWLEDGEMENTS}

The authors would like to thank all participating investigators for their contribution to the study.

Funding. Sponsorship and the journal's rapid service fee for this study were provided by Servier, Moscow, Russian Federation.

Authorship. All named authors meet the International Committee of Medical Journal Editors (ICMJE) criteria for authorship for this manuscript, take responsibility for the integrity of the work as a whole and have given final approval for the version to be published. All authors had full access to all of the data in this study and take complete responsibility for the integrity of the data and accuracy of the data analysis.

Medical Writing and Editorial Assistance. Editorial assistance to prepare this article was provided by Amanda Whereat B.Sc. independent medical writer of Speak the Speech Consulting. Support for this assistance was funded by Servier, France."

Disclosures. Maria Glezer received honoraria as scientific coordinator of this study and for lectures from Servier, Moscow, Russian Federation.

Compliance with Ethics Guidelines. All procedures complied with the ethical standards of the responsible committee on human experimentation (institutional and national), the Helsinki Declaration of 1964, as revised in 2013, and the European Independent Ethics Committee. The CHOICE-2 protocol was approved by the Inter-University Ethics Committee (protocol no. 09-14 dd. 23/10/2014; Moscow), and informed consent for inclusion in the study was obtained from all patients.

Data availability. The data sets analyzed during the current study are available from the corresponding author on reasonable request. 
Open Access. This article is licensed under a Creative Commons Attribution-NonCommercial 4.0 International License, which permits any non-commercial use, sharing, adaptation, distribution and reproduction in any medium or format, as long as you give appropriate credit to the original author(s) and the source, provide a link to the Creative Commons licence, and indicate if changes were made. The images or other third party material in this article are included in the article's Creative Commons licence, unless indicated otherwise in a credit line to the material. If material is not included in the article's Creative Commons licence and your intended use is not permitted by statutory regulation or exceeds the permitted use, you will need to obtain permission directly from the copyright holder. To view a copy of this licence, visit http://creativecommons.org/licenses/by$\mathrm{nc} / 4.0 /$.

\section{REFERENCES}

1. GBD 2016 Causes of Death Collaborators. Global, regional, and national age-sex specific mortality for 264 causes of death, 1980-2016: a systematic analysis for the Global Burden of Disease Study 2016. Lancet. 2017;390(10100):1151-210.

2. Poole-Wilson PA, Voko Z, Kirwan BA, de Brouwer S, Dunselman PH, Lubsen J. Clinical course of isolated stable angina due to coronary heart disease. Eur Heart J. 2007;28(16):1928-35.

3. Povsic TJ, Broderick S, Anstrom KJ, et al. Predictors of long-term clinical endpoints in patients with refractory angina. J Am Heart Assoc. 2015;4(2): e001287.

4. Fihn SD, Gardin JM, Abrams J, et al. 2012 ACCF/ AHA/ACP/AATS/PCNA/SCAI/STS guideline for the diagnosis and management of patients with stable ischemic heart disease: a report of the American College of Cardiology Foundation/ American Heart Association task force on practice guidelines, and the American College of Physicians, American Association for Thoracic Surgery, Preventive Cardiovascular Nurses Association, Society for Cardiovascular Angiography and Interventions, and Society of Thoracic Surgeons. Circulation. 2012;126(25):e354-471.

5. Ferrari R, Ford I, Greenlaw N, et al. Geographical variations in the prevalence and management of cardiovascular risk factors in outpatients with CAD: data from the contemporary CLARIFY registry. Eur J Prev Cardiol. 2015;22(8):1056-65.

6. Steg PG, Greenlaw N, Tendera M, et al. Prevalence of anginal symptoms and myocardial ischemia and their effect on clinical outcomes in outpatients with stable coronary artery disease: data from the International Observational CLARIFY Registry. JAMA Intern Med. 2014;174(10):1651-9.

7. Huqi A, Morrone D, Guarini G, Capozza P, Orsini E, Marzilli M (2016) Stress testing after complete and successful coronary revascularization. Can J Cardiol 32(8):986.e23-9.

8. Tonino PA, De Bruyne B, Pijls NH, et al. Fractional flow reserve versus angiography for guiding percutaneous coronary intervention. $\mathrm{N}$ Engl J Med. 2009;360(3):213-24.

9. Maron DJ, Spertus JA, Mancini GB, et al. Impact of an initial strategy of medical therapy without percutaneous coronary intervention in high-risk patients from the clinical outcomes utilizing revascularization and aggressive drug evaluation (COURAGE) trial. Am J Cardiol. 2009;104(8): 1055-62.

10. Steg PG, Ferrari R, Ford I, et al. Heart rate and use of beta-blockers in stable outpatients with coronary artery disease. PLoS ONE. 2012;7(5):e36284.

11. Kantor PF, Lucien A, Kozak R, Lopaschuk GD. The antianginal drug trimetazidine shifts cardiac energy metabolism from fatty acid oxidation to glucose oxidation by inhibiting mitochondrial long-chain 3-ketoacyl coenzyme A thiolase. Circ Res. 2000;86(5):580-8.

12. Fragasso G, Perseghin G, De Cobelli F, et al. Effects of metabolic modulation by trimetazidine on left ventricular function and phosphocreatine/adenosine triphosphate ratio in patients with heart failure. Eur Heart J. 2006;27(8):942-8.

13. Dalla-Volta S, Maraglino G, Della-Valentina P, Viena P, Desideri A. Comparison of trimetazidine with nifedipine in effort angina: a double-blind, crossover study. Cardiovasc Drugs Ther. 1990;4(Suppl 4):853-9.

14. Szwed H, Sadowski Z, Elikowski W, et al. Combination treatment in stable effort angina using trimetazidine and metoprolol. Results of a randomized, double-blind, multicentre study (TRIMPOL II). Eur Heart J. 2001;22(24):2267-74.

15. Detry JM, Sellier P, Pennaforte S, Cokkinos D, Dargie $\mathrm{H}$, Mathes $\mathrm{P}$. Trimetazidine: a new concept in the treatment of angina. Comparison with propranolol in patients with stable angina. Trimetazidine 
European Multicenter Study Group. Br J Clin Pharmacol. 1994;37(3):279-88.

16. $\mathrm{Hu} \mathrm{B}, \mathrm{Li} \mathrm{W}, \mathrm{Xu} \mathrm{T}$, Chen T, Guo J. Evaluation of trimetazidine in angina pectoris by echocardiography and radionuclide angiography: a meta-analysis of randomized, controlled trials. Clin Cardiol. 2011;34(6):395-400.

17. Danchin N, Marzilli M, Parkhomenko A, Ribeiro JP. Efficacy comparison of trimetazidine with therapeutic alternatives in stable angina pectoris: a network meta-analysis. Cardiology. 2011;120(2): 59-72.

18. Zhao $\mathrm{Y}$, Peng $\mathrm{L}$, Luo $\mathrm{Y}$, et al. Trimetazidine improves exercise tolerance in patients with ischemic heart disease: a meta-analysis. Herz. 2016;41(6):514-22.

19. Gao D, Ning N, Niu X, Hao G, Meng Z. Trimetazidine: a meta-analysis of randomised controlled trials in heart failure. Heart. 2011;97(4):278-86.

20. Zhang L, Lu Y, Jiang H, et al. Additional use of trimetazidine in patients with chronic heart failure: a meta-analysis. J Am Coll Cardiol. 2012;59(10): 913-22.

21. Zhou $X$, Chen J. Is treatment with trimetazidine beneficial in patients with chronic heart failure? PLoS ONE. 2014;9(5):e94660.

22. Lopatin YM, Rosano GM, Fragasso G, et al. Rationale and benefits of trimetazidine by acting on cardiac metabolism in heart failure. Int J Cardiol. 2016;203:909-15.

23. Michaelides AP, Spiropoulos K, Dimopoulos K, Athanasiades D, Toutouzas P. Antianginal efficacy of the combination of trimetazidine-propranolol compared with isosorbide dinitrate-propranolol in patients with stable angina. Clin Drug Invest. 1997;13(1):8-14.

24. Ruzyllo W, Szwed H, Sadowski Z, et al. Efficacy of trimetazidine in patients with recurrent angina: a subgroup analysis of the TRIMPOL II study. Curr Med Res Opin. 2004;20(9):1447-54.

25. Glezer M. Real-world evidence for the antianginal efficacy of trimetazidine from the russian observational CHOICE-2 study. Adv Ther. 2017;34(4): 915-24.

26. Kloner RA, Chaitman B. Angina and Its Management. J Cardiovasc Pharmacol Ther. 2017;22(3): 199-209.

27. Ellison KE, Gandhi G. Optimising the use of betaadrenoceptor antagonists in coronary artery disease. Drugs. 2005;65(6):787-97.
28. Sabido M, Thilo H, Guido G. Long-term effectiveness of bisoprolol in patients with angina: a realworld evidence study. Pharmacol Res. 2019;139: 106-12.

29. European Society of Cardiology (ESC). 2019 Guidelines on chronic coronary syndromes. ESC clinical practice guidelines 2019. https://www. escardio.org/Guidelines/Clinical-PracticeGuidelines/Chronic-Coronary-Syndromes.

30. Tendera M, Fox K, Ferrari R, et al. Inadequate heart rate control despite widespread use of beta-blockers in outpatients with stable CAD: findings from the international prospective CLARIFY registry. Int J Cardiol. 2014;176(1):119-24.

31. Boyle RM, Bray CL, Naqvi N, Croxson RS, Cruickshank JM. A comparison of once and twice daily atenolol for angina pectoris. Int $\mathrm{J}$ Cardiol. 1983;3(1):25-35.

32. Gislason GH, Rasmussen JN, Abildstrom SZ, et al. Long-term compliance with beta-blockers, angiotensin-converting enzyme inhibitors, and statins after acute myocardial infarction. Eur Heart J. 2006;27(10):1153-8.

33. Al-Lamee R, Thompson D, Dehbi H-M, et al. Percutaneous coronary intervention in stable angina (ORBITA): a double-blind, randomised controlled trial. Lancet. 2018;391(10115):31-40.

34. Peng S, Zhao M, Wan J, Fang Q, Fang D, Li K. The efficacy of trimetazidine on stable angina pectoris: a meta-analysis of randomized clinical trials. Int J Cardiol. 2014;177(3):780-5.

35. Boden WE, O'Rourke RA, Teo KK, et al. Optimal medical therapy with or without PCI for stable coronary disease. $\mathrm{N}$ Engl $\mathrm{J}$ Med. 2007;356(15):1503-16.

36. Weisz G, Genereux P, Iniguez A, et al. Ranolazine in patients with incomplete revascularisation after percutaneous coronary intervention (RIVER-PCI): a multicentre, randomised, double-blind, placebocontrolled trial. Lancet. 2016;387(10014):136-45.

37. Peri-Okonny PA, Patel KK, Jones PG, et al. Low diastolic blood pressure is associated with angina in patients with chronic coronary artery disease. J Am Coll Cardiol. 2018;72(11):1227-32.

38. Vidal-Petiot E, Greenlaw N, Ford I, et al. Relationships between components of blood pressure and cardiovascular events in patients with stable coronary artery disease and hypertension. Hypertension. 2018;71(1):168-76. 